\title{
Exposure to atmospheric pollutants is associated with alterations of gut microbiota in spontaneously hypertensive rats
}

\author{
DONGMEI CHEN, CHUNLING XIAO, HUANRONG JIN, BIAO YANG, \\ JIAYU NIU, SIYUAN YAN, YE SUN, YUAN ZHOU and XIANGMING WANG \\ Department of Pathogenobiology, College of Basic Medical Sciences, \\ Jilin University, Changchun, Jilin 130021, P.R. China
}

Received January 21, 2019; Accepted May 28, 2019

DOI: $10.3892 /$ etm.2019.7934

\begin{abstract}
Atmospheric particulate matter with a diameter $<2.5 \mu \mathrm{m}(\mathrm{PM} 2.5)$ and pollution are worldwide environmental problems and may have negative effects on cardiovascular disease through the lung and gut. The dynamics of intestinal microflora in response to particulate pollutants is unclear. The present study investigated changes in the gut microbiota related to pollutant exposure using spontaneously hypertensive rats (SHR). DNA was extracted from fecal samples. Amplicon Generation and the quality control of PCR products were performed. PCR products was sequenced on an Illumina HiSeq 2500 platform. Data analysis included: operational taxonomic unit (OTU) clustering and species annotation, alpha diversity, beta diversity, principal coordinates analysis (PCoA), and the use of PICRUSt bioinformatics software. The microbial diversity of the SHR rats was inversely associated with exposure to pollutants. In terms of relative abundance, 24 bacterial genera and 2 genera in particular (Actinobacillus and Fusobacterium) significantly declined, and one genus (Treponema) increased. Moreover, pollutant exposure was associated with the accumulation of genes from the gut microbiota that are implicated in cardiovascular diseases. From the long-term exposure experiment, rats appeared to respond to pollutant injury. In conclusion, these results suggest that the effects of atmospheric pollutants on organisms are not limited to the respiratory tract, but also include the gastrointestinal tract. Pollutants are likely to influence the intestinal microbiota and promote the progression of cardiovascular disease.
\end{abstract}

Correspondence to: Dr Chunling Xiao, Department of Pathogenobiology, College of Basic Medical Sciences, Jilin University, 126 Xinmin Street, Changchun, Jilin 130021, P.R. China

E-mail: xiaochunling2000@163.com; xiaochunling@symc.edu.cn

Key words: pollutant exposure, gut microbiota, PICRUSt, cardiovascular diseases

\section{Introduction}

The effect of air pollution on the human body has become an important problem in China and the focus of research. According to epidemiological data, mortality due to respiratory disease in north China significantly correlates with winter fog and haze (1). Mixed pollutants, including particulate matter (PM) and sulfide and nitrogen oxides, have become the main causes of cardiovascular diseases in winter (2). The deadliest form of air pollution during haze is atmospheric particulate matter with a diameter $<2.5 \mu \mathrm{m}$ (PM2.5) (3). PM2.5 is associated with increased morbidity and mortality from respiratory and cardiovascular diseases such as cardiac arrhythmia, congestive heart failure, and ischemic heart disease $(4,5)$.

Although most research on the links between pathology and pollution have focused on cardiovascular and respiratory effects, there are also indications that air pollutants affect the human gastrointestinal tract (6). Epidemiological studies have revealed associations between air pollution exposure and various gastrointestinal diseases, including inflammatory bowel disease (7), appendicitis (8), irritable bowel syndrome (9) and enteric infections in infants (10). A rise in total measured air pollutants was associated with an increase in hospitalizations for inflammatory bowel disease (11) and abdominal pain (3). Airborne PM2.5 in particular increases the permeability of the intestinal gut, disrupting the epithelial barrier and thereby triggering gastrointestinal disorders (11).

The gut microbiota is an important contributor to human health (12). A dysfunctional gut microbiome has been implicated in the development of many disorders, such as diabetes (13), obesity (14) and cardiovascular disease (15). A study of pollutants reported that smoking may exert pathological effects, at least in part by regulating intestinal microbiota (11).

Little is known about how air pollution influences the gut microbiome (16), but there is evidence that the gastrointestinal tract can be affected by respiratory tract exposure to PM2.5 (17). Human studies have shown that mucociliary air pollutants are cleared quickly from the lungs (18). The documented effect of air pollutants on the respiratory and gastrointestinal systems, and the effect of tobacco smoke on intestinal microbiota, supports the need for more information regarding how PM2.5 may influence the gut microbiota. 
Spontaneously hypertensive rats (SHR) have been deemed suitable for studying interactions of the digestive and respiratory systems under air pollutant exposure (11). To gain greater understanding of such interactions, in the present study we investigated alterations in the intestinal flora microbiota of rats challenged by PM2.5 exposure, using 16S rDNA sequencing.

\section{Materials and methods}

SHR rats. Specific pathogen-free SHR male rats $(\mathrm{n}=10$; aged 8-11 weeks; weight $200 \pm 10 \mathrm{~g}$ ) were purchased from Beijing Weitong Lihua Animal Technology (license number SCXK Beijing 2012-0001) and housed at $22 \pm 2^{\circ} \mathrm{C}$ and $45-55 \%$ humidity, with natural day and night hours and natural light. Food and water were provided ad libitum. Adaptive feeding was allowed for one week prior to experiments while their activity and eating was observed.

The present study was approved by the Ethics Committee of the School of Basic Medical Sciences, Jilin University (Changchun, China).

Source and treatment with PM2.5. The PM2.5 used in the present study was collected from Shenyang city atmosphere with a $120 \mathrm{~F}$ flow dust sampler (sampling flow, 1,000 l/min). A suspension of PM2.5 dust (4 mg/ml) was prepared with sterile saline solution (19).

Preparation of mixed gases. Standard mixed gas was provided by Dalian Special Gas Industry $\left(\mathrm{SO}_{2} 2013.1 \times 10^{-6} ; \mathrm{NO}_{2}\right.$ $1187.0 \times 10^{-6}$; CO $\left.23199.6 \times 10^{-6}\right)$. The cylinder filling pressure was $9.0 \mathrm{MPa}$ and the volume was 401.

Animal handling. The Institutional Animal Care and Use Committee of Shenyang Medical College approved the protocols for handling the rats. The rats were anesthetized with an inhalation of nose and mouth of chloral hydrate $(0.7 \mathrm{mg} / 100 \mathrm{~g})$ prior to each treatment with PM2.5 dust-saline.

The rats were dosed with PM2.5 dust-saline (1 $\mathrm{ml}$ of $4 \mathrm{mg} / \mathrm{ml}$ ) once per week for 12 weeks, using a non-exposed tracheal perfusion method (19). Each rat was treated individually to ensure the dosage. The rats breathed mixed gases $\left(\mathrm{SO}_{2}\right.$ $2013.1 \mathrm{mg} / \mathrm{m}^{3} ; \mathrm{NO}_{2}, 1187.0 \mathrm{mg} / \mathrm{m}^{3} ; \mathrm{CO}, 23199.6 \mathrm{mg} / \mathrm{m}^{3}$ ) by dynamic inhalation (19). The flow rate of the mixed gas was $1.2 \mathrm{l} / \mathrm{min}$, each exposure time was $3 \mathrm{~h}$.

The control group rats inhaled normal air in the cage. During the experimental period, each rat was housed individually in separate cages.

Sampling set up. Fecal samples were collected randomly from 3 rats at $0,7,15,30,60$ and 90 days, $<5 \mathrm{~g}$ fecal sample was collected from each individual.

Stool DNA extraction. A Qiagen 51504 QIAamp DNA Stool Mini kit (Qiagen $\mathrm{GmbH}$ ) was used to extract the total DNA. The quantity of the extracted DNA was determined with a NanoDrop 2000 (Thermo Fisher Scientific, Inc.).

Amplicon generation and the quality control of PCR products. The PCR primers were selected based on the sequences of the V3 and V4 hyper-variable regions of the bacterial 16S rRNA gene. The microbial V3-V4 region was amplified by PCR using the primers F: 5'-ACTCCTACGGGAGGCAGCA and R: 3'-GGACTACHVGGGTWTCTAAT (20). All PCR reactions were performed with Phusion High-Fidelity PCR Master Mix (New England Biolabs, Inc.). The quality of the PCR products was determined by electrophoresis with $2 \%$ agarose gel for detection. Samples with bright main strips between 400-450 bp were chosen for further experiments. PCR products were mixed in equidensity ratios. The mixed PCR products were purified with a Qiagen Gel Extraction kit (Qiagen).

Library preparation and sequencing process. Sequencing libraries were generated using a TruSeq DNA PCR-free sample preparation kit (Illumina, Inc.) in accordance with the manufacturer's recommendations, and index codes were added. The library quality was assessed on the Qubit 2.0 fluorometer (Thermo Fisher Scientific, Inc.). Lastly, the library was sequenced on an Illumina HiSeq 2500 platform (Illumina, Inc.) and 250-bp paired-end reads were generated.

Data analysis. The reads were merged using the FLASH tool, which merges paired-end reads from the original DNA fragments. Quality filtering on the raw tags was performed under specific filtering conditions in accordance with the QIIME (v1.9.1) quality-controlled process. Sequences that overlapped by more than $10 \mathrm{bp}$ were assembled and junk reads were discarded.

Production sequences with $\geq 97 \%$ similarity were assigned to the same operational taxonomic unit (OTU). The representative sequence for each OTU was screened for further annotation. Species annotation for each representative sequence was picked from each OTU. The Green Gene Database was used based on the RDP3 classifier phylogenetic tree. The relatedness of different OTUs, and the differences in the dominant species in different samples (groups), was conducted using MUSCLE software (version 3.8.31). OTU abundance information was normalized using a standard sequence number corresponding to the sample with the least sequences.

Alpha diversity and the community richness index were calculated with QIIME (version 1.9.1) and displayed with R software (version 2.15.3). Beta diversity of weighted and unweighted UniFrac values were calculated using QIIME (version 1.9.1). Cluster analysis was preceded by principal component analysis with the 'ggplot-2' package in the $\mathrm{R}$ software (version 2.15.3). Principal coordinates analysis (PCoA) was performed to obtain the principal coordinates and visualize the complex multidimensional data. UPGMA (unweighted pair group method with arithmetic mean) clustering was performed for hierarchical clustering to interpret the distance matrix using average linkage and was conducted using QIIME (version 1.9.1).

PICRUSt (phylogenetic investigation of communities by reconstruction of unobserved states) bioinformatics software was applied for predicting the gene family abundance of bacterial communities, based on the 16S rDNA gene data and a database of reference genomes (21). PICRUSt consisted of two steps, gene content inference and metagenome inference, performed as previously described (22). The t-test (SPSS 19.0; 
A

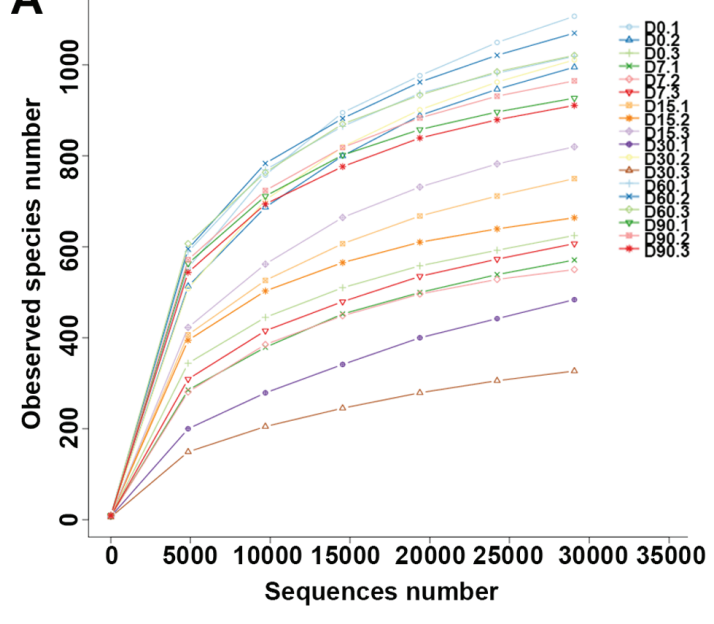

C

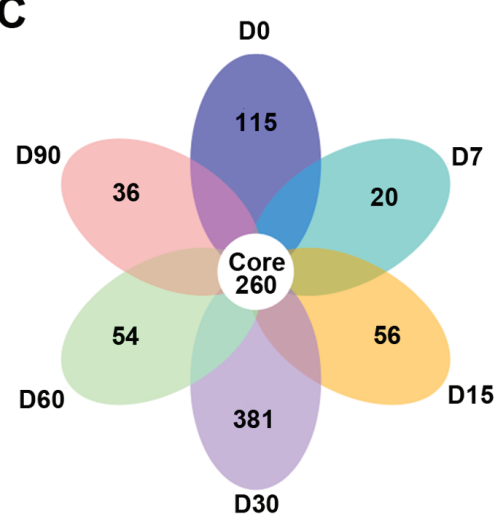

B

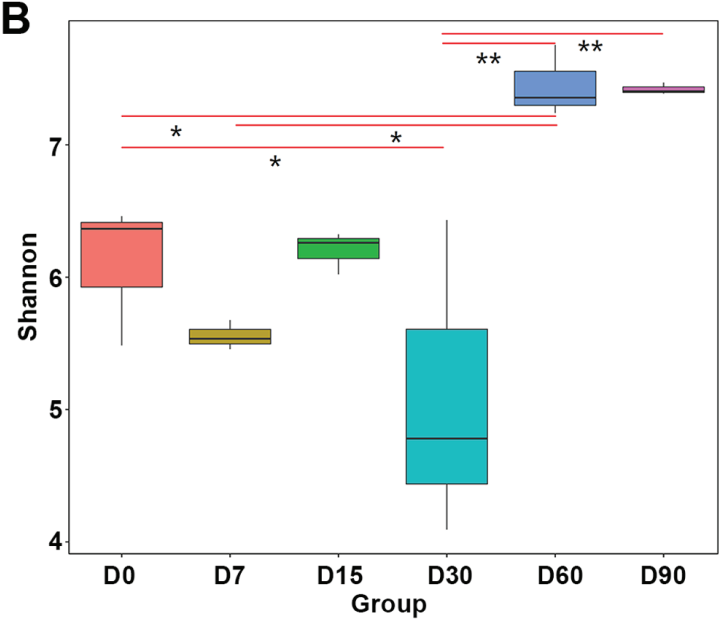

D

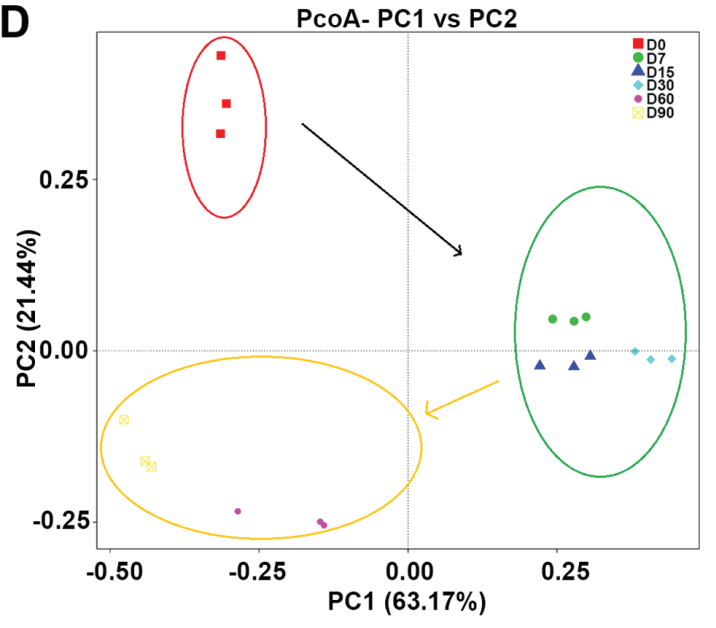

Figure 1. Different microbial diversity indices in different groups. (A) Bacterial rarefaction curves based on observed species were used to assess the depth of coverage for each sample. (B) Box-plot of grouped Shannon index, showed the variation trend of diversity. ${ }^{*} \mathrm{P}<0.05$, ${ }^{* *} \mathrm{P}<0.01$. (C) Flora data showed the number of shared OTUs and the number of independent OTUs, shared OTUs was shown in the core area, and the shared OTUs were in the petal. (D) Weighted Unifrac PCoA showed similarity among the bacterial communities associated with each sample.

Chicago, IL, USA) was used to analyse the data with normal distribution. $\mathrm{P}<0.05$ was defined as the standard criterion for statistical significance (23).

\section{Results}

Changes in the gut microbiota associated with PM2.5 exposure. To investigate changes in the gut microbiota in response to PM2.5, we performed amplicon sequencing of the fecal samples from the exposed rats at 6 sampling timepoints $(0,7$, 15, 30, 60 and 90 days). Rarefaction curves revealed no new observed species after 20,000 reads, which meant that almost all bacterial species were detected in all samples (Fig. 1A).

Based on $97 \%$ sequence similarity, all the sequences of regions V3-V4 were clustered into 10,887 bacterial OTUs. The diversity of the microbial communities was measured using Shannon diversity indices (Fig. 1B). We found that pollutant treatment was associated with these results; there was a significant reduction in bacterial diversity at 7 days. At day 60 , the bacterial diversity was higher (Shannon index $=7.45$ ) than at day 0 (Shannon index $=6.103$ ). According to the flora data (Fig. 1C), the fecal samples shared 260 different OTUs. The day-30 fecal sample was the most populous among the independent OTUs (381 independent OTUs), and the next populous was the day 0 sample (115 independent OTUs).

The beta diversity of the bacterial communities associated with rats was investigated through $\mathrm{PCoA}$, which was performed on the phylogenetic beta-diversity matrix obtained by UniFrac (Fig. 1D). The samples exhibited good repeatability. Moreover, ANOSIM of the weighted UniFrac distances revealed significant differences in the bacterial communities between groups $(\mathrm{R}=0.0689, \mathrm{P}=0.033)$.

Intestinal bacteria at the phylum level. Taxonomic assignment analysis at the phylum level is shown in Fig. 2A. After the bacterial OTU representative sequences were taxonomically classified, the results showed that the most abundant and common phyla (abundance within the community $\geq 1 \%$ ) in all samples were Firmicutes (35\%), Bacteroidetes (29\%), Proteobacteria (17\%) and Tenericutes (1\%).

Over time, the relative abundance of bacteria changed to a great extent at the phylum level (Fig. 2B-E). Statistically significant differences of the top phyla were found (compared with the day 0 sample), as follows: day 7, Firmicutes, Bacteroidetes and Tenericutes; day 15, Firmicutes, Bacteroidetes, Proteobacteria and Tenericutes; day 30, Bacteroidetes; day 60, Proteobacteria 

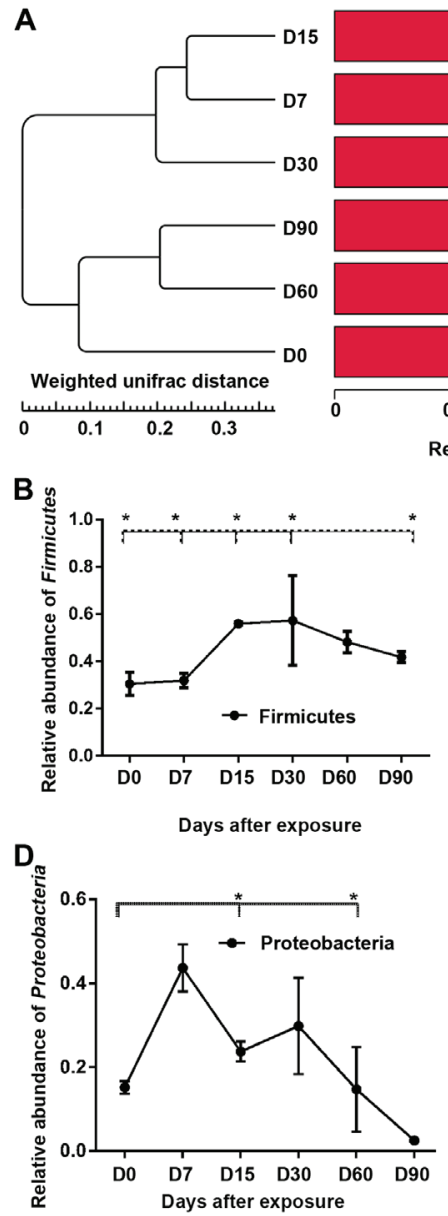
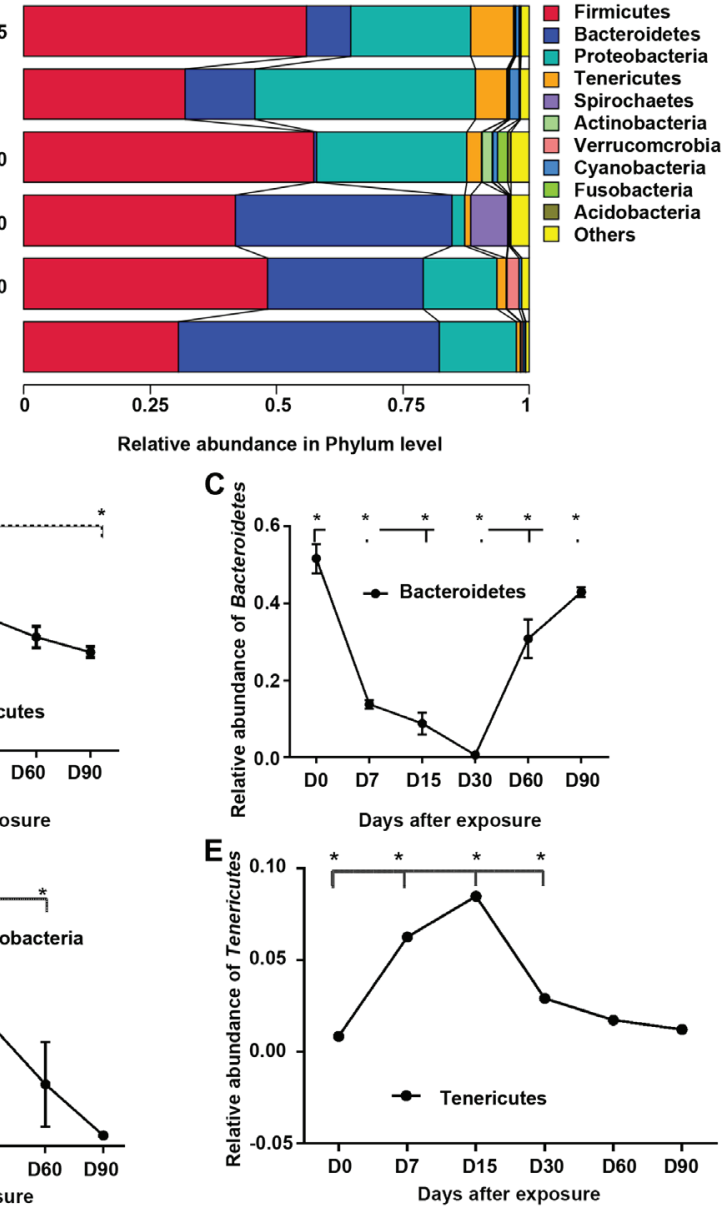

Figure 2. Main components of intestinal bacteria at the phylum level. (A) Relative abundance of the RDP-classified sequence reads at the phylum level (TOP 10). (B) Shifts and the analysis of significance in the relative abundance of Firmicutes with all samples ${ }^{*} \mathrm{P}<0.05$. (C) Shifts and the significant analysis of relative abundance of Bacteroidetes with all samples ${ }^{*} \mathrm{P}<0.05$. (D) Shifts and the significant analysis of relative abundance of Proteobacteria with all samples ${ }^{*} \mathrm{P}<0.05$. (E) The shifts and the significant analysis of relative abundance of Tenericutes with all samples ${ }^{*} \mathrm{P}<0.05$.

and Tenericutes; and day 90, Firmicutes and Bacteroidetes. Compared with the day 0 sample, after 30 days of exposure Firmicutes increased by $37 \%$ and Tenericutes increased by $44 \%$, and there were significant decreases in Bacteroidetes (-17\%) and Proteobacteria (-83\%).

Alterations in the gut bacterial compositions. To investigate further the effects of air pollutants on the intestinal microflora of SHR rats, 209 genera were identified from the gut bacterial communities of the samples. Among these, 9 abundant genera constituted $>0.1 \%$ of the total sequences in at least one sample (Fig. 3A). These were: Prevotellaceae-Prevotella (37.25\%), Bacillus (0.45\%), Veillonella (1.23\%), Achromobacter (3.11\%), Helicobacter (2.31\%), Lactobacillus (1.42\%), Campylobacter (0.59\%), Paraprevotellaceae-Prevotella (6.93\%) and Oscillospira (2.41\%).

Compared with the day- 0 sample, the most significant differences in OTUs at the genus level were the following (Fig. 3B and C): Cetobacterium, Mycoplasma, Treponema, Actinobacillus, Prevotella, Odoribacter, Achromobacter, Spironema, Fusobacterium, Campylobacter, Clostridium and Parvimonas. The result showed that two genera (Actinobacillus and Fusobacterium) significantly decreased after 30 days. However, the bacterial community showed significant increases in relative abundance of only one genus (Treponema). During 30 days of incubation, other bacterial genera showed dramatic fluctuations in abundance but, no changes were observed after 30 days.

Functional maturation of the gut bacterial community and the shifts of disease-involved genes in gut metagenomics. To investigate how pollutants affect the functional profile of gut microbiota, PICRUSt analysis was used to analyse the KEGG pathway compositions in bacterial populations (Fig. 4A). The result suggested that most predicted genes were enriched in the following pathways: organismal systems, cellular processes, environmental information processing, human diseases, genetic information processing and metabolism.

The changes in most genes from treatment groups of SHR rats did not correlate with the time-points (Fig. 4B). Changes in gut function or genes in the SHR rats were not linear after prolonged exposure to the pollutants. Genes related to human diseases increased significantly (23\%) prior to 30 days (Fig. 4C). Surprisingly, the genes related to human diseases significantly decreased after long-term pollutant exposure (15\% lower at day 90 compared with that of day 0 ).

The percentage of genes linked to cardiovascular diseases peaked at day 15 (Fig. 4D). Moreover, the genes detected before 


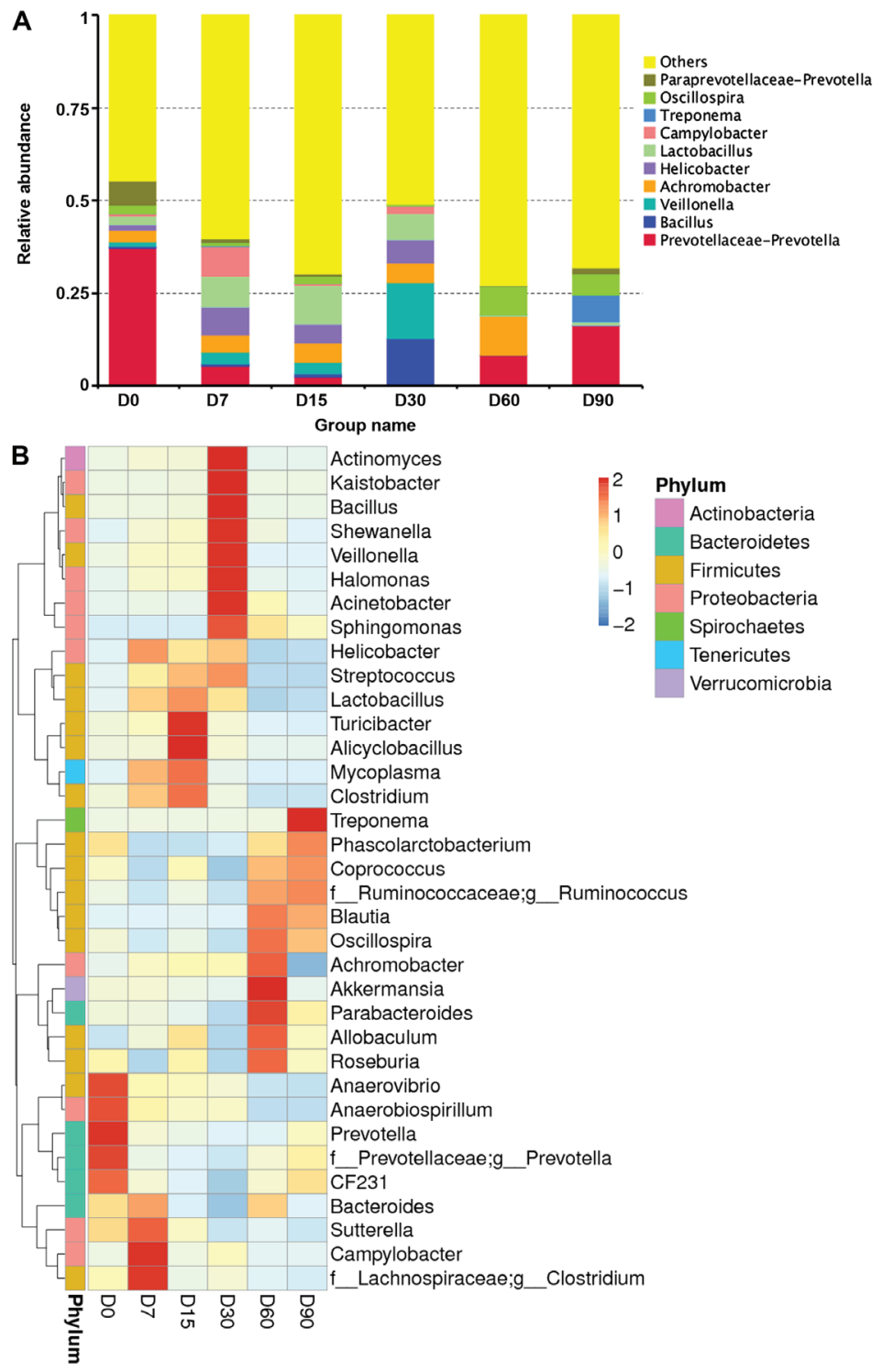

Figure 3. Significant alterations in the gut bacterial compositions. (A) Relative abundance and the average proportional distributions of gut bacterial Top 10 genus identified in all groups. (B) Heat map and hierarchical clustering of genera in the gut bacterial communities of all samples. The color of the squares on the left indicate the average abundance of the OTU in each group. The OTUs are ordered via phylogenetic positions.

day 15 were all significantly higher than the baseline (day 0), and then decreased after 15 days. At day 90 , the percentage of genes related to cardiovascular disease was significantly lower than that at day 0 .

Most genes were annotated and enriched to the environmental information-processing pathway (Fig. 4E). Compared with that of day 0 , the enrichment of related genes increased significantly at day 7, 15, 30, 60 and 90 (Fig. 4E).

\section{Discussion}

In the present study, we investigated shifts in gut microbial populations in SHR rats within 90 days of continuous exposure to PM2.5 air pollutant. The results revealed changes in gut microbiota composition and functional adaptation of the gut bacterial community associated with long-term pollutant exposure.

A recent study showed that Bacteroidaceae, Oscillospira, and Ruminococcus are the most abundant genera in the guts of
Wistar rats reared under normal conditions (24). By contrast, the present study found that Prevotellaceae-Prevotella, Paraprevotellaceae-Prevotella and Achromobacter were the most abundant. The gut microbiota structure of the SHR rat is different from those of Wistar rats (1). In previous studies, PM2.5 was thought to affect the microbial community in the gut after going through the digestive tract (25). In this study, we found a significant decrease in alpha diversity in the gut bacterial community associated with exposure to air pollutants. To some extent, damaging effects of air pollutants on gut microbial diversity were found in animal experiments.

In the present study, after long-term exposure the restoration of intestinal microbial diversity indicated that the intestinal system of rats has a certain ability to maintain microbial diversity. Whether humans have such rapid recovery is unknown. The decrease in diversity during the early days (0-15 days) of exposure indicated gut diseases and simplification of metabolic types. This matches previous epidemiological investigation results, in which PM could 

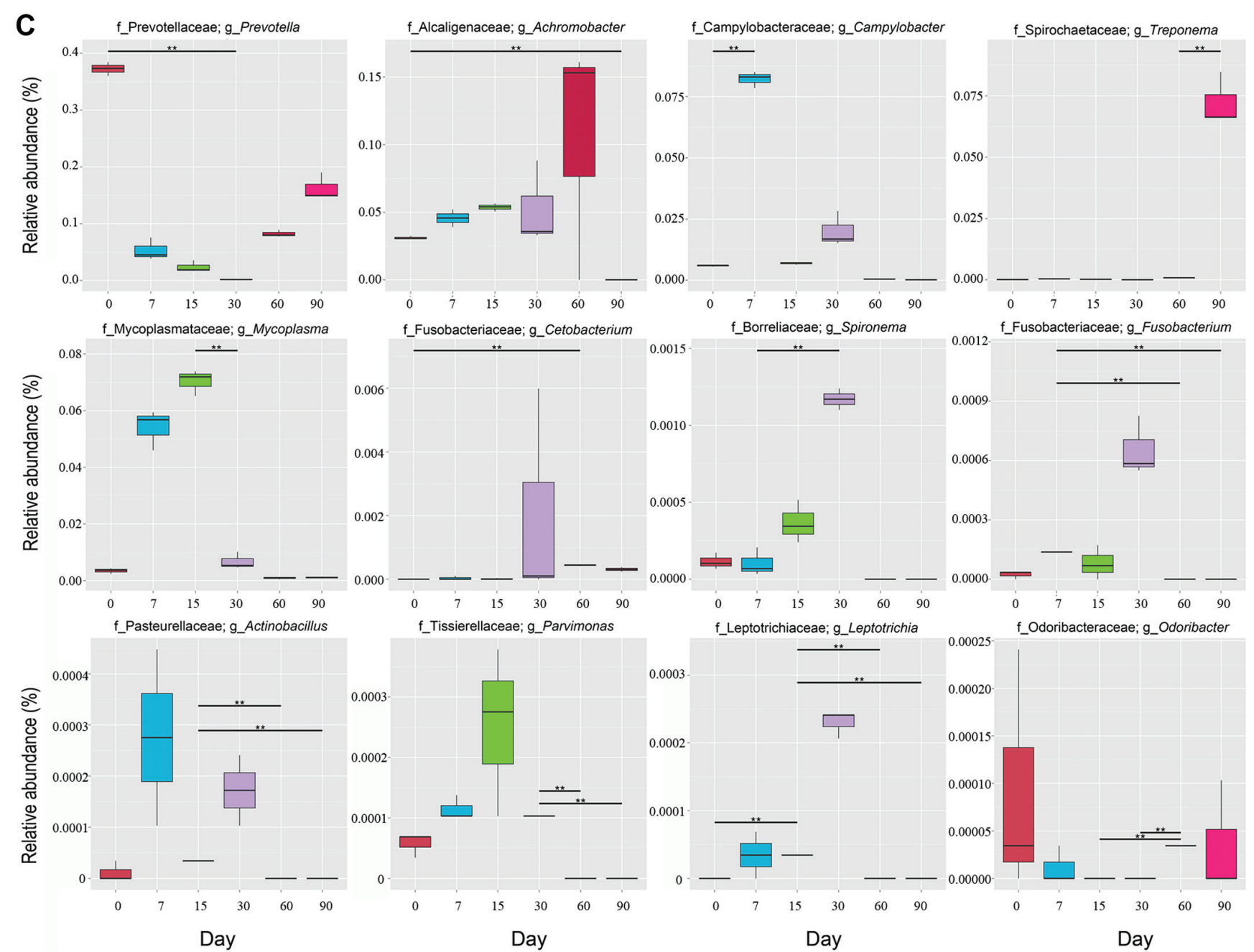

Figure 3. Continued. (C) MetaStat analysis showing the most differential OTUs (genus level) from all samples. Extreme significant difference that was obtained between the samples is indicated; $\left({ }^{* *} \mathrm{P}<0.01\right)$.

alter the gut microbiome and result in gut disease (6). How this process was triggered and whether it is related to the influence of PM2.5 on microbiota colonization in the gastrointestinal tract (26) remains unknown. In particular, in the present study the change in gut microbes in the SHR rats was characterized by a major transition from Bacteroidetes to Firmicutes under pollutant exposure (Fig. 2A). Our results showed significant decline in the relative abundances of 2 phyla (Bacteroidetes and Spirochaetea) after 90 days of long-term exposure, and the number of phyla did not significantly increase. In the present study, pollutant exposure did not affect the structure of the phyla in the SHR rats, but the quantitative changes were significant and responded rapidly to pollutant exposure.

At the genus level, Cetobacterium, Mycoplasma, Treponema, Actinobacillus, Prevotella and Odoribacter were the genera that declined most significantly after 90 days (>5\% decline). Mycoplasma and Treponema are specific pathogens that are associated with chronic respiratory infections (28). These pathogens that are common in the respiratory tract, which changed dramatically in the intestinal tract, and the mechanism of this change is unclear. Moreover, the flora of the lungs and intestines may interchange and colonize via the lymphatic system (25). Our experiments seem to confirm this finding.

Prevotella is a newly discovered strain in a recently isolated genus that includes 20 species (16), with the most common species being a black pigmented strain (P. melaninogenica). This genus is mainly concentrated in the healthy human oral cavity, female genital tract (29). It is a common opportunistic pathogen in the clinic and can cause endogenous infections, especially of the female genital tract and oral cavity (29). Thus, Actinobacillus may be a candidate pathogen of the host upper respiratory tract, digestive tract, and urogenital tract; it belongs to thousands of normal flora. In the present study, the change in the abundance of Prevotella may be related to the oral perfusion of the pollutants.

Odoribacter was reported as enriched in mice with colorectal cancer and may be related to tumor development (30). Genera of the bacterial phyla Cetobacterium may be related to digestive tract function. Cetobacterium is associated with the biosynthesis of acetic acid (31).

It is reported that intestinal microbiota metabolism of choline/phosphatidylcholine produces trimethylamine (TMA), which is further metabolized to a proatherogenic species, trimethylamine- $N$-oxide (TMAO) (15). TMAO is 
A

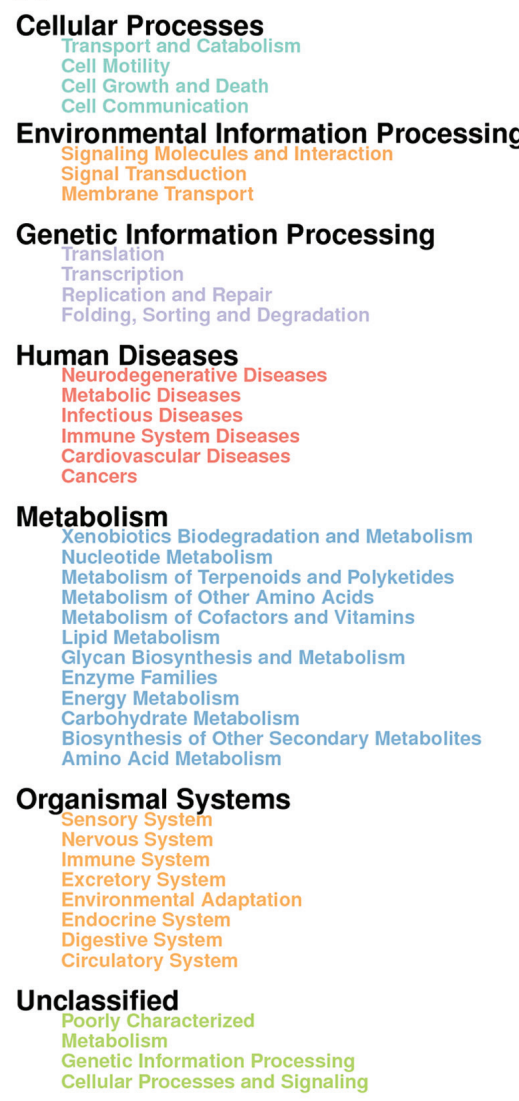

\section{KEGG pathway annotation}

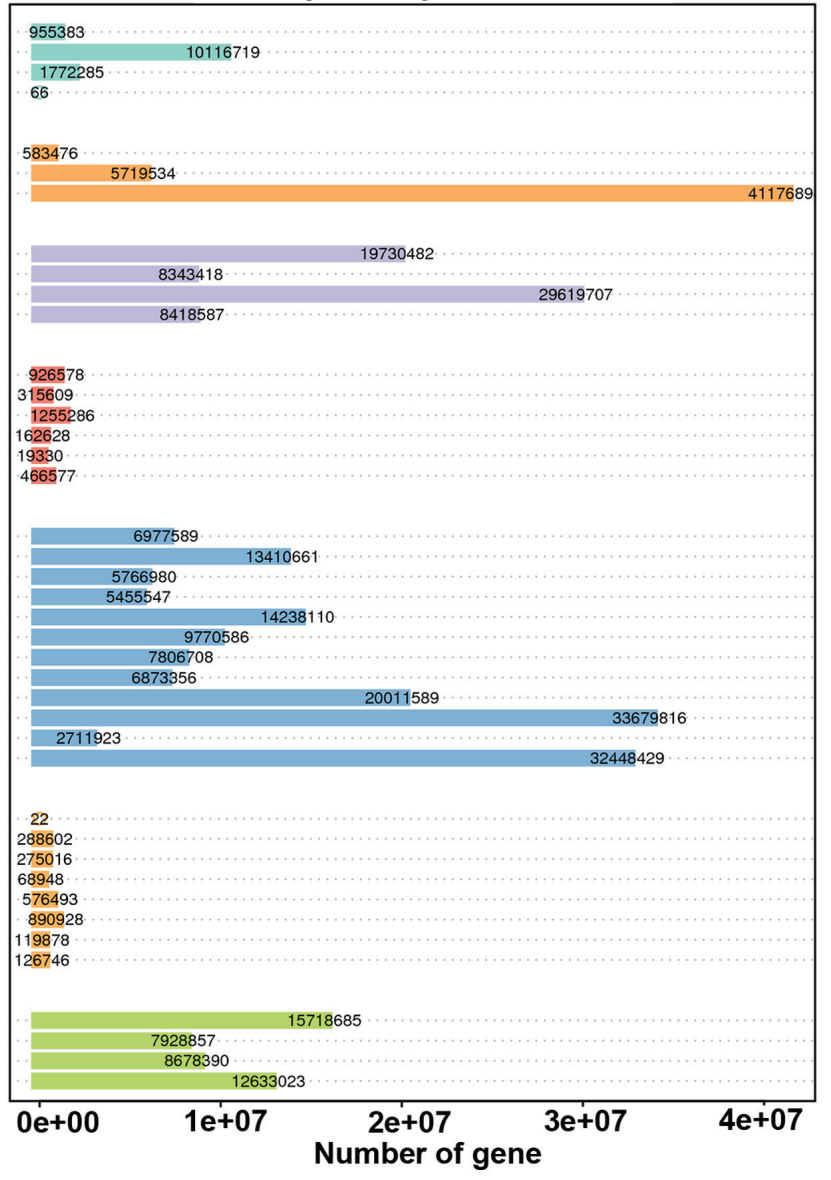

B
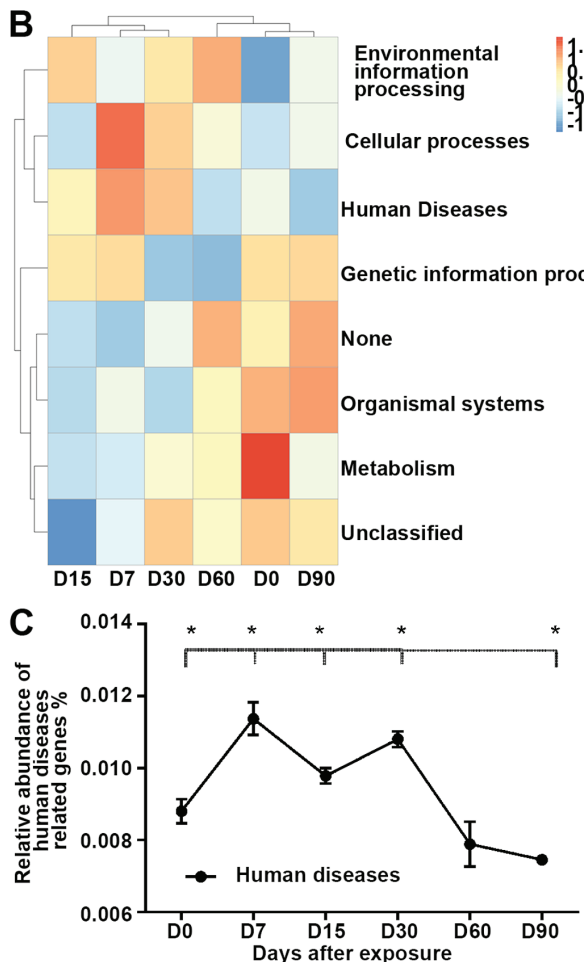

D

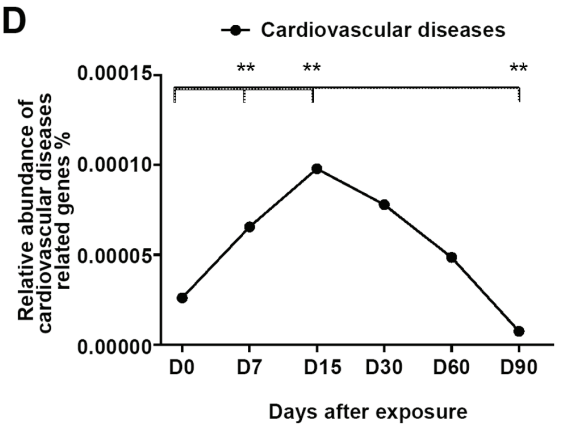

E

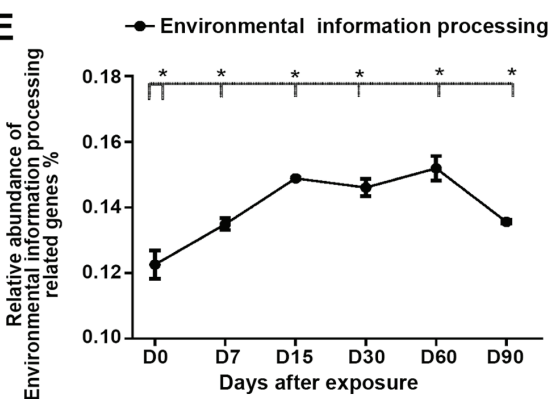

Figure 4. KEGG pathway annotation and the quantitative distribution of the gene enrichment. (A) Overview of the predicted data. (B) Shifts in gut bacterial functional profiles as the pollutant treated SHR rats. Heat map and hierarchical clustering of differentially abundant KEGG pathways identified at 6 sampled time-points $(0,7,15,30,60$ and 90 days). The values of color in the heat map represent the normalized relative abundance of KEGG pathways (log 10). Heat map and hierarchical clustering of differentially abundant KEGG pathways identified at 6 sampled time-points (0, 7, 15, 30, 60 and 90 days). The values of color in the heat map represent the normalized relative abundance of KEGG path. (C-E) Analysis was performed to identify the significantly differentially abundant of selected pathways (human diseases, cardiovascular diseases, and environmental information processing) among groups and day 0 sample. Asterisks indicate the significant differences that were obtained between D0 sample and samples of following observational days $\left({ }^{*} 0.01<\mathrm{P}<0.05\right.$, $\left.^{* *} \mathrm{P}<0.01\right)$. 
closely related to the increased occurrence of major adverse events of cardiovascular diseases. The bacterial community and its composition provide different TMAOs (32). Increased TMAO levels will significantly affect systemic cholesterol accumulation, leading to increased generation of atherosclerotic plaque (33).

The gut microbiota have also been proven to be involved in the anabolism of TMAO as the producer of the precursor (TMA) (34). Microecology studies have shown that higher TMAO plasma concentrations were associated with the Prevotella enterotype, as opposed to the Bacteroides enterotype (34). In the present study, from investigation at the genera level we found that Prevotellaceae-Prevotella (relative abundance $37.25 \%$ ) is the most enriched genus in all samples. Moreover, pollutant exposure reduced the abundance of this population within 30 days (Fig. 3C). This may be related to the intestinal microbial background of SHR rats (24), the relative abundance of Bacteroides was decreased after treatment with pollutant (Fig. 3A). This may suggest that a metabolic disorder of TMAO leads to an increased risk of cardiovascular disease at the microbial level.

PICRUSt is a closed-access analysis based on an established database. It is not comprehensive, but can provide accurate directional guidance. In this study, the rat intestinal metagenome responded to pollutant exposure within 30 days (Fig. 4). After 30 days, the intestinal metagenome showed an ability to repair damage. The accumulation of cardiovascular disease-related genes in the gut showed a statistically significant association with air pollutants. This is consistent with other reports (33). Previous studies have shown that, in response to changes in environmental factors, the changes in microbial functional diversity were greater than changes in system diversity (35). The evidence from metagenomic studies may be more useful for clinical studies than that of microbial systematic investigations.

In conclusion, herein the effects of air pollutants on the gut microbiota of SHR rats are reported. Moreover, statistically significant changes in the microbiota were investigated. Changes in phylum levels indicate that the intake of air pollutants highly affects the lower taxa, and abnormalities in metabolism and nutrient absorption may be triggered by the intake of air contaminants. The pathological features of these changes require further investigation. The results of this study support that the consequences and destruction of air pollutants on the microbial structure of the intestinal tract are no less than that of the respiratory system.

\section{Acknowledgements}

We would like to thank the undergraduate students of SYMC: Gang Li, Yuting Deng and Guotong Zhao for taking care of the rats, and Jiamiao Wang, Xin Chen and Sun Rui for the pollutant exposure experiment.

\section{Funding}

The present study was funded by the National Natural Science Foundation of China (grant no. 30872083), the Shenyang Science and Technology Public Welfare Research Project (4190070101) and the Shenyang Science and Technology
Project (grant nos. F13-221-9-36 and F14-181-1-00) by the Science and Technology Bureau of Shenyang.

\section{Availability of data and materials}

The datasets used and/or analyzed during the present study are available from the corresponding author on reasonable request.

\section{Authors' contributions}

DC wrote the manuscript. CX and HJ helped with the animal handling. DC, BY and JN contributed to DNA extraction and PCR. SY and YS were responsible for the data collection and analysis. YZ and XW were in charge of the library preparation and sequencing process. The final version was read and adopted by all the authors.

\section{Ethics approval and consent to participate}

The present study was approved by the Ethics Committee of the School of Basic Medical Sciences, Jilin University (Changchun, China).

\section{Patient consent for publication}

Not applicable.

\section{Competing interests}

The authors declare that they have no competing interests.

\section{References}

1. Ma M, Li S, Jin H, Zhang Y, Xu J, Chen D, Kuimin C, Yuan Z and Xiao C: Characteristics and oxidative stress on rats and traffic policemen of ambient fine particulate matter from Shenyang. Sci Total Environ 526: 110-115, 2015.

2. Stockfelt L, Andersson EM, Molnár P, Gidhagen L, Segersson D, Rosengren A, Barregard L and Sallsten G: Long-term effects of total and source-specific particulate air pollution on incident cardiovascular disease in Gothenburg, Sweden. Environ Res 158: 61-71, 2017

3. Chen H, Goldberg MS and Villeneuve PJ: A systematic review of the relation between long-term exposure to ambient air pollution and chronic diseases. Rev Environ Health 23: 243-297, 2008.

4. Chen YC, Weng YH, Chiu YW and Yang CY: Short-term effects of coarse particulate matter on hospital admissions for cardiovascular diseases: A case-crossover study in a tropical city. J Toxicol Environ Health A 78: 1241-1253, 2015.

5. Bhalla DK: Ozone-induced lung inflammation and mucosal barrier disruption: Toxicology, mechanisms, and implications. J Toxicol Environ Health B Crit Rev 2: 31-86, 1999.

6. Salim SY, Kaplan GG and Madsen KL: Air pollution effects on the gut microbiota: A link between exposure and inflammatory disease. Gut Microbes 5: 215-219, 2014.

7. Zuo T, Kamm MA, Colombel JF and Ng SC: Urbanization and the gut microbiota in health and inflammatory bowel disease. Nat Rev Gastroenterol Hepatol 15: 440-452, 2018.

8. Sekirov I, Russell SL, Antunes LC and Finlay BB: Gut microbiota in health and disease. Physiol Rev 90: 859-904, 2010.

9. Shouval DS and Rufo PA: The role of environmental factors in the pathogenesis of inflammatory bowel diseases: A Review. JAMA Pediatr 171: 999-1005, 2017.

10. Calkins BM: A meta-analysis of the role of smoking in inflammatory bowel disease. Dig Dis Sci 34: 1841-1854, 1989.

11. Mutlu EA, Engen PA, Soberanes S, Urich D, Forsyth CB, Nigdelioglu R, Chiarella SE, Radigan KA, Gonzalez A, Jakate S, et al: Particulate matter air pollution causes oxidant-mediated increase in gut permeability in mice. Part Fibre Toxicol 8: 19,2011. 
12. Kinross JM, Darzi AW and Nicholson JK: Gut microbiome-host interactions in health and disease. Genome Med 3: 14, 2011.

13. Barton W, Penney NC, Cronin O, Garcia-Perez I, Molloy MG, Holmes E, Shanahan F, Cotter PD and O'Sullivan O: The microbiome of professional athletes differs from that of more sedentary subjects in composition and particularly at the functional metabolic level. Gut 67: 625-633, 2018.

14. Kowalski TJ, Liu SM, Leibel RL and Chua SC Jr: Transgenic complementation of leptin-receptor deficiency. I. Rescue of the obesity/diabetes phenotype of LEPR-null mice expressing a LEPR-B transgene. Diabetes 50: 425-435, 2001.

15. Tang WH and Hazen SL: The gut microbiome and its role in cardiovascular diseases. Circulation 135: 1008-1010, 2017.

16. Man WH, de Steenhuijsen Piters WA and Bogaert D: The microbiota of the respiratory tract: Gatekeeper to respiratory health. Nat Rev Microbiol 15: 259-270, 2017.

17. Hernández AF and Tsatsakis AM: Human exposure to chemical mixtures: Challenges for the integration of toxicology with epidemiology data in risk assessment. Food Chem Toxicol 103: 188-193, 2017.

18. Moller W, Haussinger K, Winkler-Heil R, Stahlhofen W, Meyer T, Hofmann W and Heyder J: Mucociliary and long-term particle clearance in the airways of healthy nonsmoker subjects. J Appl Physiol (1985) 97: 2200-2206, 2004.

19. Xiao C, Li S, Zhou W, Shang D, Zhao S, Zhu X, Chen K and Wang R: The effect of air pollutants on the microecology of the respiratory tract of rats. Environ Toxicol Pharmacol 36: 588-594, 2013.

20. Ma Y, Ding S, Liu G, Fang J, Yan W, Duraipandiyan V, Al-Dhabi NA, Esmail GA and Jiang H: Egg protein transferrin-derived peptides IRW and IQW regulate citrobacter rodentium-induced, inflammation-related microbial and metabolomic profiles. Front Microbiol 10: 643, 2019.

21. Catry E, Bindels LB, Tailleux A, Lestavel S, Neyrinck AM, Goossens JF, Lobysheva I, Plovier H, Essaghir A, Demoulin JB, et al: Targeting the gut microbiota with inulin-type fructans: Preclinical demonstration of a novel approach in the management of endothelial dysfunction. Gut 67: 271-283, 2018.

22. Henning SM, Yang J, Shao P, Lee RP, Huang J, Ly A, Hsu M, Lu QY, Thames G, Heber D, et al: Health benefit of vegetable/ fruit juice-based diet: Role of microbiome. Sci Rep 7: 2167, 2017

23. Yang Y, Deng Y and Cao L: Characterising the interspecific variations and convergence of gut microbiota in Anseriformes herbivores at wintering areas. Sci Rep 6: 32655, 2016.
24. Shatzkes K, Tang C, Singleton E, Shukla S, Zuena M, Gupta S, Dharani S, Rinaggio J, Connell ND and Kadouri DE: Effect of predatory bacteria on the gut bacterial microbiota in rats. Sci Rep 7: 43483, 2017.

25. Chakradhar S: A curious connection: Teasing apart the link between gut microbes and lung disease. Nat Med 23: 402-404, 2017.

26. Barrett KE and Wu GD: Influence of the microbiota on host physiology - moving beyond the gut. J Physiol 595: 433-435, 2017.

27. Arrieta MC, Bistritz L and Meddings JB: Alterations in intestinal permeability. Gut 55: 1512-1520, 2006.

28. Mortaz E, Adcock IM, Folkerts G, Barnes PJ, Paul Vos A and Garssen J: Probiotics in the management of lung diseases. Mediators Inflamm 2013: 751068, 2013.

29. Wang J, Gao Y and Zhao F: Phage-bacteria interaction network in human oral microbiome. Environ Microbiol 18: 2143-2158, 2016.

30. Viaud S, Saccheri F, Mignot G, Yamazaki T, Daillère R, Hannani D, Enot DP, Pfirschke C, Engblom C, Pittet MJ, et al: The intestinal microbiota modulates the anticancer immune effects of cyclophosphamide. Science 342: 971-976, 2013.

31. De Vadder F, Kovatcheva-Datchary P, Goncalves D, Vinera J, Zitoun C, Duchampt A, Bäckhed F and Mithieux G: Microbiotagenerated metabolites promote metabolic benefits via gut-brain neural circuits. Cell 156: 84-96, 2014

32. Tang WH, Wang Z, Levison BS, Koeth RA, Britt EB, Fu X, Wu Y and Hazen SL: Intestinal microbial metabolism of phosphatidylcholine and cardiovascular risk. N Engl J Med 368: 1575-1584, 2013.

33. Koeth RA, Wang Z, Levison BS, Buffa JA, Org E, Sheehy BT, Britt EB, Fu X, Wu Y, Li L, et al: Intestinal microbiota metabolism of L-carnitine, a nutrient in red meat, promotes atherosclerosis. Nat Med 19: 576-585, 2013

34. Tang WH, Kitai T and Hazen SL: Gut microbiota in cardiovascular health and disease. Circ Res 120: 1183-1196, 2017.

35. Kostovcik M, Bateman CC, Kolarik M, Stelinski LL, Jordal BH and Hulcr J: The ambrosia symbiosis is specific in some species and promiscuousinothers: Evidencefromcommunity pyrosequencing. ISM E J 9: cc) (i) $\mathrm{E}$ This work is licensed under a Creative Commons Attribution-NonCommercial-NoDerivatives 4.0 International (CC BY-NC-ND 4.0) License. 